\title{
Essais
}

Revue interdisciplinaire d'Humanités

Hors-série 7 | 2022

Récits d'outre-thèse

\section{Récit d'une préparation et concrétisation d'une expérience de recherche}

The story of a preparation and realisation of a Research Experience

\section{Aubin Gonzalez}

\section{(2) OpenEdition}

Journals

Édition électronique

URL : https://journals.openedition.org/essais/10872

DOI : 10.4000 /essais. 10872

ISSN : 2276-0970

Éditeur

École doctorale Montaigne Humanités

\section{Édition imprimée}

Date de publication : 15 avril 2022

ISBN : 979-10-970024-00-0

ISSN : $2417-4211$

Référence électronique

Aubin Gonzalez, «Récit d'une préparation et concrétisation d'une expérience de recherche », Essais [En ligne], Hors-série 7 | 2022, mis en ligne le 01 février 2022, consulté le 18 janvier 2023. URL : http:// journals.openedition.org/essais/10872 ; DOI : https://doi.org/10.4000/essais.10872

Ce document a été généré automatiquement le 18 janvier 2023.

Tous droits réservés 


\title{
Récit d'une préparation et concrétisation d'une expérience de recherche
}

\author{
The story of a preparation and realisation of a Research Experience
}

\section{Aubin Gonzalez}

Tout de suite, ce point capital : je ne raconte pas ma vie. Il est convenu que je n'exhiberai dans cette ego-histoire qu'une part de moi. L'egolaborator, si l'on veut, ou bien l'ego-faber. Parce que je ne parle pas de peinture, par exemple, de théâtre ni de musique, parce que je ne dis rien de

ceux que j'aime, il est bien évident qu'ici

l'essentiel est tu'1.

Georges Duby

1 En guise de propos liminaire à la présentation de mon travail de thèse de doctorat en 2019, soutenu publiquement devant de la famille, des amis et des collègues, j'ai affirmé que l'exercice de la soutenance est d'abord celui de la fin d'une expérience de recherche et d'enseignement dans un milieu professionnel et académique. Aujourd'hui jeune maître de conférences heureux à l'Université Bordeaux Montaigne, j'enseigne l'Histoire contemporaine au sein du département d'Histoire et je suis depuis septembre 2020 membre du Centre d'Études des Mondes Modernes et Contemporains.

2 Lorsque j'ai été sollicité pour apporter ma contribution à ce numéro intitulé « Récits d'outre-thèse » j'ai naturellement répondu présent. Au-delà du simple fait de revenir sur un jeune parcours de recherche et d'en présenter les perspectives actuelles, cette contribution me permet d'exprimer ma profonde reconnaissance envers différents compagnons de route. Qu'il s'agisse de mes anciens et actuels collègues enseignantschercheurs confirmés, de mes collègues agents administratifs ou techniques, je voudrais remercier ici toutes celles et ceux qui m'ont soutenu durant tout mon 
parcours universitaire et à qui je dois tant. En écrivant ces pages je songe aussi à mon directeur de thèse, François Godicheau, qui dans les moments de doutes scientifiques ou personnels, a toujours répondu présent et c'est la raison pour laquelle il est également destinataire de cette contribution.

3 Étant jeune chercheur, je me dois de vous avertir : je n'ai pas du tout l'ambition, ni la prétention, d'imiter les travaux d'égo-histoire à laquelle tant de chercheurs confirmés et respectés de mon champ de recherche se sont déjà pliés. Vis-à-vis de ceux qui sont entrés dans la profession d'historien dix ans plus tôt, je n'ai sans doute pas assez de recul sur une expérience de recherche dont je conçois qu'elle puisse paraître jeune (c'est l'évidence même) et maladroite. Dans les pages qui suivent, j'espère davantage me plier à un exercice d'humilité scientifique.

Après être brièvement revenu sur mon parcours et sur la mise au point de l'objet de mes recherches, j'en exposerai ensuite les principaux résultats, les principaux apports et les prolongements envisagés pour de futurs travaux que j'espère mener.

\section{À la recherche des secrets de famille : élaboration de l'objet de recherche et parcours universitaire}

4 Les premières pages de l'histoire de mon parcours universitaire ont commencé à s'écrire ici, en septembre 2010, au sein de ce qui s'appelait encore Université Michel de Montaigne - Bordeaux III. Jeune étudiant en première année de master galvanisé par la recherche autour de ses origines familiales et d'un premier séminaire de troisième année de Licence sur les débats historiographiques de la Guerre civile animé par Monsieur François Godicheau, je poussai la porte de deux séminaires de recherches. Le premier était intitulé «introduction à la pensée politique italienne» tandis que le second proposait de s'intéresser à l'exil des républicains espagnols en France. Si le premier m'a permis de faire mes premiers pas en épistémologie sous la bienveillance de Monsieur Sandro Landi, auprès de qui j'ai découvert des auteurs tels que Quentin Skinner ${ }^{2}$, Emile Benveniste ${ }^{3}$ ou Léo Strauss ${ }^{4}$, c'est néanmoins le séminaire consacré à l'exil qui a été déterminant pour la suite ${ }^{5}$.

5 J'y ai retrouvé Monsieur François Godicheau avec bonheur mais j'éprouvais un manque, comme un vide dans le récit sur lequel on réfléchissait. Partir de l'exil de 1939 jusqu'en 1945 et après? L'histoire ne pouvait pas s'arrêter là ! D'après l'écho de ma propre histoire familiale, je ne descends pas d'exilés républicains, mes grands-parents n'ont pas participé à la résistance contre l'occupant nazi. Eux étaient des immigrés espagnols venus s'installer en France dans l'espoir d'y trouver de meilleures conditions de vie. Il m'était pourtant difficile de ne pas m'interroger sur l'existence d'une suite entre leur parcours migratoire débuté vers la moitié des années cinquante et l'existence d'une dictature féroce stigmatisant toute forme d'opposition ou de mécontentement.

6 Ayant accepté le défi lancé par le Professeur Godicheau, qui m'avait alors proposé d'engager un travail de fin de séminaire sur le sujet, j'avais rendu un premier exposé qui m’a ensuite permis de préparer un mémoire de deuxième année de Master. Intitulé "L'émigration sous le franquisme: étude des politiques et parcours migratoires (1950-1970), ce mémoire était une critique de la littérature scientifique sur le sujet ${ }^{6}$. Une sorte de porte d'entrée s'ouvrait dans un champ de recherche dont je m'attachais à connaître les bases, mais aussi les apports récents, aussi bien du côté français que du côté espagnol, avec les conseils bienveillants de chercheurs et chercheuses catalans 
confirmés lors de mon séjour ERASMUS au sein de l'Universitat Autònoma de Barcelona et du Centre d'Estudis sobre Dictadures i Democràcies (CEDID) entre septembre 2011 et février 2012. À l'issue de ce mémoire, le terrain défriché m'avait permis de constater qu'il existait bien des points de contact remettant en cause le cloisonnement des générations d'Espagnols installées en France depuis 1939 en deux vagues de migrations a priori parfaitement distinctes.

7 Entre ce qui demeure aujourd'hui un «état de l'art ", et l'objet d'étude que j'ai travaillé pour le doctorat, le fossé me paraît immense. Pourtant, ce sont là deux éléments d'une seule et même démarche de réflexion, laquelle a pu être prolongée grâce aux prémices de ce qui est aujourd'hui une véritable politique d'insertion professionnelle menée par l'Université Bordeaux-Montaigne. En effet, au terme de mon Master, l'idée de poursuivre l'enquête que j'avais commencé à mener m'enthousiasmait car j'avais perçu un prolongement à ma question de départ. Dès le mois de septembre 2012, j'ai eu l'honneur de bénéficier d'un Contrat doctoral dévolu aux étudiants en situation de handicap par le ministère de l'Enseignement Supérieur et de la Recherche ${ }^{7}$. Je me suis donc inscrit en première année de doctorat avec un projet de thèse qui avait alors pour titre: «Les Ouvriers catholiques dans l'émigration sous le franquisme: une attitude entre conformisme et détachement $»$.

Il s'agissait de continuer à repenser le phénomène de l'émigration espagnole sous le franquisme en se centrant plus particulièrement sur les groupes d'individus qui s'affichaient comme catholiques, entre 1950 et 1975. J'espérais ainsi expliquer le silence historiographique sur l'engagement explicite des paroisses dans une opposition politique au régime dictée par un esprit de réelle concurrence avec les groupes issus de l'exil républicain de 1939.

Animé par l'enthousiasme d'enquêter sur un objet inattendu, j'ai alors entrepris de faire entendre les voix de ces immigrés à partir d'une campagne de témoignage essentiellement réalisée dans la région bordelaise.

Entre les journées passées un dans périmètre d'enquête compris entre le quartier SaintMichel et le Cours de l'Yser et étendu à quelques communes limitrophes de Bordeaux, j'ai également choisi de cibler particulièrement d'anciens paroissiens du Solar Español de Bordeaux. Fondée en 1920 par deux pères jésuites français, la mission catholique du Solar Español de Bordeaux est effectivement l'un des hauts lieux de la présence espagnole à Bordeaux, et qui subsiste encore aujourd'hui ${ }^{8}$. Le choix d'un tel terrain d'enquête s'est imposé à moi en raison de contraintes logistique indéniables me concernant, mais aussi d'une histoire commune : la région bordelaise et plus largement le Sud-Ouest de la France ont toujours constitué une zone stratégique aussi bien pour les groupes issus de l'exil républicain de 1939 que pour ceux qui étaient pleinement soutenus par la dictature.

10 À l'écoute et à la transcription de récits qui m'étaient livrés volontiers, par des témoins heureux de pouvoir raconter leur parcours migratoire tout en s'interrogeant sur l'utilité de leur témoignage, une impérieuse nécessité m'est apparue. Parmi les témoignages recueillis auprès des paroissiens du Solar Español, certains insistaient volontiers sur l'expression d'une sorte de solidarité intergénérationnelle, expression qui résonnait étrangement avec ce que j'avais repéré lors de la préparation de mon mémoire de master.

11 Il fallait donc poursuivre la réflexion sur l'émigration espagnole et ses catégories de recherche et repenser la morphologie de la présence espagnole installée en France 
entre 1950 et 1970. Pour ce faire, j'ai dû reprendre la chronologie du récit que j'entendais proposer. Il s'agissait d'éviter le piège consistant à scinder a priori un seul et même phénomène en deux vagues migratoires successives et cohérentes : d'un côté une immigration espagnole dont la présence est motivée par les conditions d'expression politiques dans l'Espagne franquiste, de l'autre une immigration dont la présence n'est pas nécessairement liée aux inquiétudes politiques mais davantage aux conditions de survie matérielles.

12 J'ai donc fait le choix de prendre pour point de départ les groupes et institutions prorépublicains et pro-franquiste en activité sur le sol français dès $1939^{\circ}$. Les lectures, les rencontres mais aussi le dépouillement d'une ample documentation d'archives en France, en Espagne et au Vatican, m'ont encouragé à recentrer mes recherches sur les institutions plutôt que sur les acteurs eux-mêmes ${ }^{10}$. Loin de décrire un ensemble de groupes et d'institutions homogènes de part et d'autre, cette radiographie de la présence espagnole décrit plutôt un large panel d'organisations et d'institutions ayant chacune des points communs et des objectifs politiques et culturels propres. Qu'est-ce qui différencie l'activité de l'exil basque, pourtant catholique, de l'activité des missions catholiques espagnoles en 1939 ? Qu'est-ce qui différencie cette activité de l'exil basque de celle menée des communistes ? Voilà quelques exemples d'interrogations auxquelles j'ai tenté de répondre, notamment dans les deux premiers chapitres. Les réponses à apporter m'ont conduit à une certaine prudence vis-à-vis du langage employé dans les études migratoires.

13 C'est pourquoi le travail que je présente ici ne correspond que partiellement à l'intitulé de départ. En introduisant la notion de réseaux et celle de " groupes de pressions », j'ai tenté de prendre de la distance par rapport aux récits produits par les « milieux » pro et anti-franquistes. Ces derniers ne sont que de simples prolongements d'une métaphore qui participe à enfermer l'étude de l'immigration espagnole en France dans des catégories selon moi réductrices. Conscient des limites de ce travail quant à la distance qui a pu être prise avec la métaphore biologique du « milieu », je pense avoir amené certains éléments intéressants pour repenser les pratiques et discours culturels de réseaux. Loin d'être innocents, ces discours et pratiques culturelles sont des marqueurs d'identification et d'expression étatique, syndicale et politique des groupes étudiés et de leur évolution de 1939 à 1976.

\section{Mise en œuvre d'une thèse : la culture catholique au cœur des mobilisations en direction de l'Espagne franquiste}

À l'issue de cette longue réflexion, mes questionnements pouvaient se résumer ainsi :

- Qui sont les groupes pro et anti-franquistes installés en France entre 1939 et 1976 ? Sont-ils exclusivement espagnols? Quelles sont les tensions qui les habitent à la lumière des différents contextes politiques dans l'Espagne, en France?

- Pourquoi s'engagent-t-ils dans l'encadrement des groupes d'immigrés arrivés massivement en France à partir de la deuxième moitié des années 50 ? Qu'est-ce qui différencie l'encadrement proposé par les organisations de classes de celui proposé par le syndicalisme catholique, celui des missions catholiques pleinement soutenues par le régime? 
-Existe-t-il une concurrence réelle entre les réseaux catholiques et ceux appuyés par les organisations de classe dans l'encadrement des Espagnols installés en France entre 1939 et 1976 ? Plus particulièrement, entre les missions soutenues par le régime et les organisations de classe? Entre les organisations de classe et le syndicalisme catholique français ou espagnol ? Entre les missions catholiques et le syndicalisme catholique?

Pour répondre à toutes ces questions, j'ai d'abord tenté de rédiger un premier jet d'introduction à la fin de l'été 2015. Abandonnée au bout de seulement quelques mois, on aurait tort de croire que cette tentative fut vaine. Elle m'a placé devant une obligation à mon sens inhérente à la recherche; celle de faire des choix dans les axes à développer ou ceux qui, au contraire, méritaient une place plus discrète dans la rédaction de mes travaux. Ce n'est qu'après en avoir discuté avec mon directeur de recherche que j'ai choisi de prendre pour point de départ une chronologie allant de 1939 à 1976 et de me lancer directement dans la rédaction du premier chapitre.

Le choix de cette chronologie, qui prend pour point de départ la fin de la Guerre civile et la mobilisation des premiers relais de solidarité en direction d'une Espagne plongée dans un conflit entre nacionales et républicains, ne doit rien au hasard. Il m'a permis de proposer un récit historiographique sur la troisième vague d'immigration capable de rendre compte de l'évolution des formes de mobilisations et d'encadrement mis en œuvre en direction de la présence espagnole installée en France jusqu'en 1976, année où la France restreint considérablement sa politique migratoire et où l'Espagne amorce sa transition vers la démocratie.

17 J'ai ainsi consacré les deux premiers chapitres de cette étude à une cartographie des mobilisations transnationales des groupes pro et antifranquistes à destination des Espagnols installés en France entre 1939 et 1950. Ces premières pages vont bien au-delà de la simple identification des institutions et groupes politiques, syndicaux ou étatiques dont la plupart ont une étiquette politique directement héritée de celle alors en jeu durant la guerre civile. Je me suis attaché à réfléchir aux modalités de négociations mises en œuvre, et à leur objet, par tous les réseaux qui œuvrent à destination des Espagnols en France. Les trois derniers chapitres constituent, quant à eux, le cœur de ma thèse. Articulés autour des négociations concernant la participation des groupes et institutions espagnols dans la France des Trente Glorieuses, dans un contexte où le pays achève de normaliser ses relations diplomatiques avec sa voisine franquiste, ces chapitres ne sauraient trouver tout leur sens sans la lecture des deux précédents. Pour raconter comment les groupes d'immigrés affichant une culture catholique, en particulier ceux proches du syndicalisme catholique, sont effectivement parvenus à proposer des marqueurs d'identification catholiques pensés comme une alternative aux valeurs du Nacional-catolicismo, il faut savoir d'où partent ces propositions et connaître le contexte dans lequel elles émergent. C'est la raison pour laquelle j'ai choisi de raconter l'histoire des solidarités antifranquistes affichant une culture catholique en France à la lumière de l'évolution du contexte politique, social et économique dans l'Espagne franquiste.

18 Convaincu que ces mêmes contextes ont favorisé l'émergence de nouvelles formes de subversion "concurrentielles » face à un régime qui abandonne progressivement ses traits fascisants pour celui d'un régime essentiellement et d'abord catholique, je suis effectivement convaincu que ces nouvelles formes de subversion ont trouvé leur prolongement au sein des groupes d'Espagnols installés en France. En racontant l'évolution des formes de solidarités mises en œuvre par les réseaux et les groupes de 
pression catholiques à destination des groupes d'immigrés installés en France entre 1939 et 1976, cette thèse raconte aussi une histoire des groupes catholiques sous le franquisme.

\section{Une vie d'écriture après la thèse}

19 Au-delà d'un travail dont les conclusions viennent enrichir l'histoire de la troisième vague d'immigration espagnole en France en situant notamment le rôle joué par le syndicalisme catholique français dans l'émergence d'une culture antifranquiste noncommuniste et démocratique, la préparation de mon doctorat me permet désormais d'envisager plusieurs perspectives de recherche.

La première d'entre elles s'inscrit directement dans le cadre du CEMMC que j'ai eu l'honneur d'intégrer en septembre 2020. Il s'agit de prolonger les discussions historiographiques du séminaire d'histoire moderne franco-espagnol à travers la proposition d'une série de conférences d'Histoire contemporaine qui réunissent des collègues français et espagnols. Dans cet esprit, plusieurs thèmes d'étude sont envisagés tels que la perception de la transition démocratique espagnole depuis la France et/ou les collaborations intergouvernementales franco-espagnoles. On pourrait également aborder la coopération économique franco-espagnole durant la dictature franquiste ou la transition démocratique espagnole, ou bien encore l'activité militante des groupes politiques espagnols en Espagne et en France ainsi que la manière dont elle dialogue avec des acteurs institutionnels des deux pays.

21 Je travaille également sur l'étude de la presse militante à destination des groupes d'immigrés espagnols non affiliés au réseau d'organisation issu des partis communistes traditionnels. Débutée durant la période de chômage qui a suivi la fin de mon doctorat, cette perspective d'étude est conçue comme un prolongement possible de la thèse présentée en décembre 2019. Elle permet de réfléchir plus avant sur les (auto)représentations des groupes d'immigrés.

22 Ainsi, dans le cadre d'une journée d'études de jeunes chercheurs organisé en avril 2021 par le laboratoire Identités, Cultures, Territoires (ICT) ${ }^{11}$ consacré aux différentes stratégies d'encadrements proposées à destination des groupes d'immigrés espagnols installés en France durant les Trente Glorieuses, j'ai parlé de l'action de la Jeunesse Ouvrière Chrétienne française en faveur de ces derniers. Cette communication fait l'objet d'un article à paraitre en février $2022^{12}$.

En outre, dans un contexte où la figure du travailleur immigré devient une figure de représentation au service de certaines solidarités non seulement pensées comme internationales mais aussi ouvrières, la parole de l'immigration deviendrait l'expression d'une véritable stratégie politique déployée par certaines organisations issues ou influencées par la "Nouvelle Gauche", au sein desquelles certains groupes catholiques ont joué un rôle important. Ces réflexions sont en train d'aboutir à un article autour du journal Frente Obrero, publié par la Federación Exterior du Frente de Liberación Popular entre 1962 et 1965 actuellement en cours d'écriture. 


\section{NOTES}

1. Maurice Agulhon et al., Essais d'ego-histoire, Paris (France), Gallimard, 1987, p. 109-110.

2. Quentin Skinner, Jerome Grossman et Jean-Yves Pouilloux, Les fondements de la pensée politique moderne, Paris, Albin Michel, 2001.

3. Émile Benveniste et Jean Lallot, Le vocabulaire des institutions indo-européennes, Paris, Minuit, 1969.

4. Leo Strauss et Olivier Sedeyn, La Persécution et l'art d'écrire, Paris, Presses Pocket, 1989.

5. Ce séminaire intitulé « Exils en France entre 1936 et 1939 » fut donné pour la première fois au premier semestre de l'année universitaire 2010-2011. Parmi toutes les problématiques abordées, figurait la participation des groupes d'exilés espagnols dans la résistance française, les exils des populations non combattantes à travers l'exemple des enfants basques, etc. Ce séminaire fut également l'occasion de faire connaissance avec plusieurs spécialistes bordelais des exils espagnols.

6. Aubin Gonzalez, L'Émigration sous le franquisme: étude sur les politiques et parcours migratoires (1950-1970), Université Bordeaux 3, Pessac, 2012, 166 p.

7. Cette thèse aurait certainement pris une toute autre tournure en l'absence de tels dispositifs. À ce titre, je remercie chaleureusement le ministère ainsi que Mesdames Violaine Lafourcade et Pauline Pinto, qui ont une large part dans la mise en œuvre de ce contrat, ainsi que dans les conditions de travail et d'études faites aux étudiantes, étudiants et collègues en situation de handicap.

8. El Solar Español de Burdeos (Francia): su origen, su labor, sus frutos y esperanzas. Testimonios favorables., Madrid, Tipografía Catolica de Alberto Fontana, 1927 ; Maria Santos-Sainz et François Guillemeteaud, Les Espagnols à Bordeaux et en Aquitaine, Bordeaux, France, Éditions Sud Ouest, 2006, $350 \mathrm{p}$.

9. Dans ce sens, j'ai notamment repris les travaux de Celia Keren. Voir Célia Keren, « Négocier l'aide humanitaire : les évacuations d'enfants espagnols vers la France pendant la guerre civile (1936-1939)", Revue d'histoire de l'enfance "irrégulière». Le Temps de l'histoire, 30 octobre 2013, $\mathrm{n}^{\circ}$ 15, p. 167-183 ; Célia Keren, L'évacuation et l'accueil des enfants espagnols en France: cartographie d'une mobilisation transnationale (1936-1940), Thèse de doctorat, École des hautes études en sciences sociales, Paris, France, 2014, 802 p.

10. Aubin Gonzalez, Les Réseaux culturels et groupes de pression catholiques de l'immigration espagnole en France (1939-1976), Université Bordeaux Montaigne, Pessac, 2019, p. 6-7.

11. "Apôtres et ouvriers: les jeunes travailleurs espagnols au sein de la JOC française (1959-1968) », communication dans le cadre de la journée d'études, Les modalités d'organisation des Espagnols en France (1920-1990), Natacha Lillo (dir.), laboratoire Identités, Cultures, Terrtoires (ICT), Université de Paris, 9 avril 2021, Paris.

12. "Nos frères espagnols ": solidarité et mobilisation jociste en France durant les années soixante, in, Matériaux pour l'histoire de notre Temps, La Contemporaine (ed.) février 2022, Nanterre (à paraître). 


\section{AUTEUR}

\section{AUBIN GONZALEZ}

Maître de conférences en Histoire Contemporaine - CEMMC (EA 2958)

Université Bordeaux Montaigne 